\title{
Bexarotene inhibits the viability of non-small cell lung cancer cells via slc10a2/ PPARY/PTEN/mTOR signaling pathway
}

\author{
Xinghao $\mathrm{Ai}^{1,2+}$, Feng $\mathrm{Mao}^{2 \dagger}$, Shengping Shen ${ }^{2}$, Yang Shentu${ }^{2}$, Jiejun Wang ${ }^{1 *}$ and Shun $\mathrm{Lu}^{2^{*}}$
}

\begin{abstract}
Background: Thirty to $40 \%$ of non-small cell lung cancer (NSCLC) patients developed higher hypertriglyceridemia in the process of treatment with bexarotene. And bioinformatics studies discovered that the expression of slc10a2 was increased in high-grade hypertriglyceridemia patients. So, we will explore the mechanism which may involve in this process.
\end{abstract}

Methods: We constructed slc10a2 overexpressed A549 cells and H1299 cells as cell models, normal A549 cells and H1299 cells as control. Then we explored the effects of slc10a2 on A549 cells and H1299 cells behaviors, including proliferation, invasion and apoptosis. The expression of apoptotic related genes and anti-cancer genes also been detected.

Results: We found that the proliferation and migration were inhibited and the apoptosis of NSCLC cells was accelerated by bexarotene. In addition, overexpressed slc10a2 in NSCLC cells can further suppress the proliferation and migration, and promote apoptosis under the treatment of bexarotene. On the contrary, the opposite results were obtained after slc10a2 gene was silenced in NSCLC cells treated with bexarotene. Moreover, the expression of caspase 3, caspase 7, PTEN, P21, P53, LKB1, TSC2 were increased and the expression of BCl-2, cyclin D1, c-FLIP were declined in NSCLC cells and slc10a2 overexpressed NSCLC cells with the treatment of bexarotene, and the opposite situations were seen after slc10a2 gene was silenced in NSCLC cells. The further studies revealed the increased expression of slc10a2 activated the expression of peroxisome proliferator-activated receptor $\gamma$ (PPARY), then up-regulated PTEN expression and down-regulated mTOR expression.

Conclusion: These results suggest that bexarotene inhibits the viability of lung cancer cells via slc10a2/PPARY/ PTEN/mTOR signaling pathway.

Keywords: Non-small cell lung cancer, A549 cells, H1299 cells, Bexarotene, slc10a2, PPARy

\section{Background}

The incidence of lung cancer is rapidly increasing in the world, and it has become the first leading cause of cancer death, especially in China [1]. Non-small cell lung cancer (NSCLC) is the most common type of lung cancer, accounting for almost $80 \%$ [2]. In clinic trials, bexarotene showed both satisfactory safety and promising efficacy for the treatment of advanced NSCLC patients [3, 4]. However $30-40 \%$

\footnotetext{
*Correspondence: jiejunw@csco.org.cn; shun_lu@hotmail.com

${ }^{\dagger}$ Equal contributors

'Department of Medical Oncology, Changzheng Hospital, The Second Military Medical University, Shanghai 200433, China

${ }^{2}$ Lung Tumor Clinical Medical Center, Shanghai Chest Hospital, Shanghai Jiao Tong University, Shanghai 200030, China
}

of the patients appeared to be more sensitive to bexarotene treatment and developed higher hypertriglyceridemia. Interestingly, survival analysis in high-grade hypertriglyceridemia patients revealed significantly longer survival compared to the patients in the control, low-grade hypertriglyceridemia and middle-grade hypertriglyceridemia groups $[5,6]$.

Bexarotene (Scheme 1) is a synthetic retinoid modulator of retinoid X receptors (RXRs), it can selectively bind and activate RXRs [2], which include (RXR $\alpha, R X R \beta$, and RXR $\gamma$ ) [7], and play a critical role in cellular growth modulation, activation of apoptosis, induction of differentiation. It has been widely explored as potential target for cancer therapies for several years $[8,9]$. The expression of RXRs was 


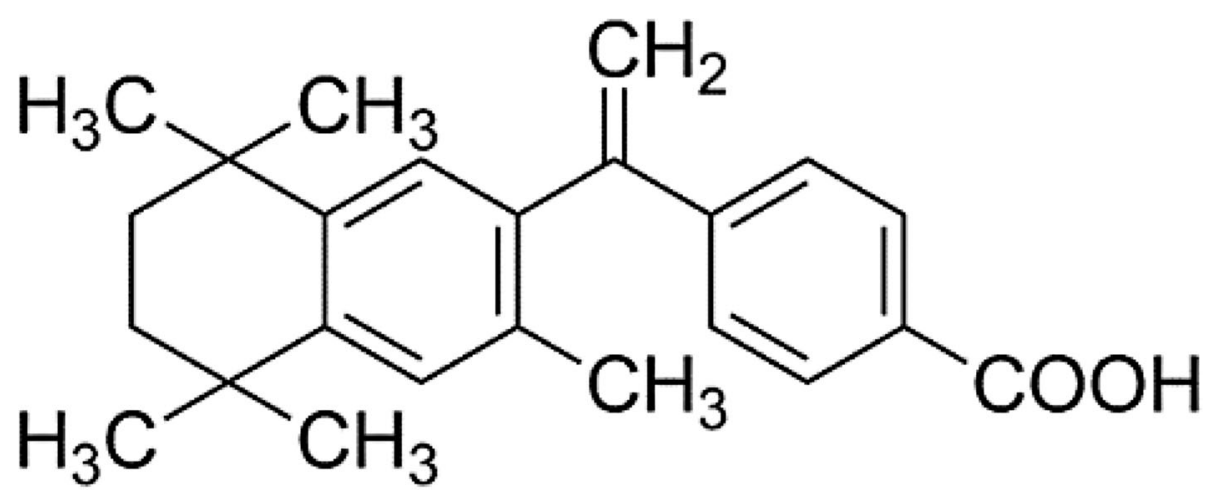

Scheme 1 The chemical structure of bexarotene

reduced in some NSCLC biopsy specimens, and increased RXRs expression has been associated with an increased survival in NSCLC patients [10].

Slc10a2 is a member of solute carrier family 10 of the sodium/bile acid co-transporter apical sodium-dependent bile acid transporter (ABST) [11], which plays a key role in the enterohepatic circulation through its reabsorption of bile acids from the ileum and indirectly conduces to cholesterol homoeostasis [12,13]. ASBT is able to inhibit the concentration of plasma triglyceride and increase the concentration of HDL (high-density lipoprotein) cholesterol [14], and now it has aroused much concern as a drug target for the pharmacological treatment of hypercholesterolaemia $[15,16]$.

The goal of this study is to explore the role of slc10a2 in the treatment of NSCLC with bexarotene. We hypothesis that bexarotene inhibits the viability of NSCLC cells (e.g. A549 cells and H1299 cells) via increasing the expression of slc10a2. In this study, we have successfully constructed slc10a2 overexpressed A549 cells and H1299 cells, and the proliferation, apoptosis, migration behaviors were detected in slc10a2 overexpressed A549 cells and H1299 cells respectively. Moreover, we also explored the expression of apoptosis genes, anti-apoptosis genes, tumor suppressor genes in slc10a2 overexpressed A549 cells and H1299 cells. Furthermore, the possible mechanism which involved in this process was discovered.

\section{Methods}

\section{Materials}

Bexarotene was obtained from Aladdin (Shanghai, China). Cell Counting Kit-8 was ordered from Dojindo (Japan). Cell culture plates and Transwell plates were ordered from Corning (NY, USA). Crystal violet was obtained from Beyotime (Haimen, China). Annexin V/fluorescein isothiocyanate (FITC) apoptosis detection kit was obtained from Beyotime biotech company (China). Gentamicin, Fetal bovine serum, glutamine, and RPMI 1640 medium were purchased from Thermo Fisher Scientific (Waltham, MA, USA). The primary antibodies including slc10a2,
PPAR $\gamma$, mTOR and PTEN (Abcam, Cambridge, UK), GAPDH (Thermo, Walteham, Washington, USA). The pcDNA3 and pcDNA3-slc10a2 plasmid, slc10a2-shRNA, GW9662 were obtained from Shanghai Funeng Biological Technology, Co., LTD.

\section{Cell lines}

The human NSCLC cell lines A549 cells (CRM-CCL$\left.185^{\mathrm{rm}}\right)$ and H1299 cells (CRL-5803 $\left.{ }^{\mathrm{ma}}\right)$ was obtained from American Type Culture Collection (Rockville, MD). Cells were maintained in RPMI 1640 media plus 10\% fetal bovine serum, $1 \%$ glutamine and $0.05 \mathrm{mg} / \mathrm{ml}$ gentamycin sulfate at $37{ }^{\circ} \mathrm{C}$ and $5 \% \mathrm{CO}_{2}$.

\section{The construction of slc10a2 overexpressed A549 cells and H1299 cells}

Before transfection, A549 cells and H1299 cells were seeded into 6-well plate at a density of $2 \times 10^{6}$ cells/well, after cells grow above $80 \%$ areas, A549 cells were transfected with $8 \mathrm{mg}$ pcDNA3-slc10a2 plasmid using Lipofect2000 transfection Reagent according to manufacturer's instructions, pcDNA3 treatment as control. The transfection medium was replaced by regular growth medium after $5 \mathrm{~h}$ transfection, and at each time point, the cells were used to observation using inverted fluorescence microscope.

\section{Proliferation assay}

A549 cells, H1299 cells and slc10a2 overexpressed A549 cells, H1299 cells were seeded in a 96-well plate, $5 \times 10^{3}$ cells per well. After $12 \mathrm{~h}$ culture, the cultured medium was replaced by conditional medium, which was added with bexarotene, bexarotene in combination with slc10a2shRNA, bexarotene in combination with GW9662 respectively. At indicated time point, ten microliter CCK-8 was added each well and continually incubated for $4 \mathrm{~h}$. Then the optical density (OD) value $(450 \mathrm{~nm})$ was determined by an enzyme-linked immunosorbent assay plate reader (Bioreader). 


\section{Transwell migration assay}

A549 cells and slc10a2 overexpressed A549 cells were starved for $12 \mathrm{~h}$, then resuspended in serum-free medium, and adjusted to $1 \times 10^{6}$ cells $/ \mathrm{ml}$. One hundred microliters of A549cells or slc10a2 overexpressed A549 cells were placed in the upper chamber of Transwell plates. Serum-free RPMI 1640 medium with bexarotene, bexarotene in combination with slc10a2-shRNA, was added to the lower chamber respectively, RPMI 1640 medium as control. Prior to the addition of cells suspension, preheated serum free RPMI 1640 medium $(300 \mu \mathrm{l})$ was added to the upper chamber. After $24 \mathrm{~h}$ incubation, the invaded cells were collected from lower chambers, then stained with crystal violet.

\section{Apoptosis assay}

The apoptosis of A549 cells, H1299 cells and slc10a2 overexpressed A549 cells, H1299 cells was analyzed using the Apoptosis Detection Kit according to the manufacturer's instructions. Cells were seeded in 6-well plate $\left(1 \times 10^{5}\right.$ cells/well $)$ with different medium, including RPMI 1640 medium plus bexarotene, RPMI 1640 medium plus bexarotene in combination with slc10a2shRNA for 2 days. At indicated times, cells were digested then resuspended in $300 \mu \mathrm{L}$ binding buffer solution which containing $5 \mathrm{ul}$ Annexin V-FITC and $5 \mathrm{ul} \mathrm{PI}$ solution, then incubated in the dark for $20 \mathrm{~min}$ at room temperature. Finally flow cytometry (FACScan; BD Biosciences) was used to analyzed the apoptotic rate of each kind of cells.

\section{RT quantitative-PCR analysis}

The total RNA of A549 cells and H1299 cells were isolated by using RNeasy kit according to the manufacturer's protocol (Qiagen, Valencia, CA). Briefly, total RNA $(1 \mu \mathrm{g})$ was used as a template to prepare cDNA (Invitrogen), and was amplified by Platinum SYBR Green qPCR SuperMix-UDG (Invitrogen). A master mix was prepared for each PCR reaction, which included Platinum SYBR Green qPCR SuperMix-UDG, forward primer, reverse primer, and $10 \mathrm{ng}$ of template cDNA. PCR was performed with the following thermocycling conditions: An initial $5 \mathrm{~min}$ at $95^{\circ} \mathrm{C}$, followed by 40 cycles of $95{ }^{\circ} \mathrm{C}$ for $30 \mathrm{~s}, 55{ }^{\circ} \mathrm{C}$ for $30 \mathrm{~s}$ and $72{ }^{\circ} \mathrm{C}$ for $30 \mathrm{~s}$. [17] The forward and backward primer sequences for Bcl-2 was 5'-CCGATCAGTGGAGCTGAAGAA-3' (sense) and 5'-GCCACAGGATGTTCTCGTCA-3' (antisense), cyclin D1:5'-CAAGGCCTGAACCTGAGGAG-3'(sense) and 5 '-CTTGGGGTCCATGTTCTGCT-3' (antisense), c-FLIP :5'-GAGTGCCGGCTATTGGACTT-3'(sense) and 5'-G CGCTTCTCTCCTACACCTC-3' (antisense), Caspase-3:5'GCGGTTGTAGAAGTTAATAAAGGT-3' (sense) and 5'TACCAGACCGAGATGTCATTCC-3' (antisense), Caspase7:5'-CGTGGGAACGGCAGGAAGT-3'(sense) and 5'-CG
GGTGGTCTTGATGGATCG-3' (antisense), PTEN:5'-CAGGATACGCGCTCGGC-3'(sense) and 5'-TCAGGAGAAGC CGAGGAAGA-3'(antisense), P21:5'-AGTCAGTTCCTTG TGGAGCC-3' (sense) and 5'-CATTAGCGCATCACAGT CGC-3'(antisense), P53:5'-GTGCTCAAGACTGGCGCTA AA-3'(sense) and 5'-CAGTCTGGCCAATCCAGGGAAG3'(antisense), LKB1:5'-GACCTGCTGAAAGGGATGCT3'(sense) and 5'-GACCTGCTGAAAGGGATGCT-3' (antisense), TSC2:5'-TCTGAACATGTGGTCCGCAG-3'(sense) and 5'-TCTGAACATGTGGTCCGCAG-3' (antisense).

\section{Western blot}

A549 cells and H1299 cells were treated with $1 \mathrm{mM}$, $5 \mathrm{mM}, 10 \mathrm{mM}$ bexarotene for $24 \mathrm{~h}$, then the cells were harvested, then total protein from tissue or cell was extracted using radioimmunoprecipitation lysis buffer containing $1 \mathrm{mM}$ phenylmethanesulfonylfluoride and the protein concentration was determined using the Bradford method (Beyotime Institute of Biotechnology, Nantong, China) according to the manufacturer's instructions. Proteins $(20 \mu \mathrm{g})$ were separated by $10 \%$ SDSPAGE and transferred onto a nitrocellulose membrane. After blocking with $5 \%$ non-fat milk for $1 \mathrm{~h}$ at $4{ }^{\circ} \mathrm{C}$, then membrane was incubated with the primary antibody at $4{ }^{\circ} \mathrm{C}$ overnight. Membranes were washed 3 times with $0.25 \%$ PBST and then incubated with the peroxidase-conjugated secondary antibody for $2 \mathrm{~h}$ at room temperature. After washed 3 times, the specific protein bands were detected using the enhanced chemiluminescence reagents [18].

\section{Statistical analysis}

All data were expressed as mean \pm S.D. Differences between the groups were analyzed using one-way analysis of variance (ANOVA) using SPSS 13.0 (SPSS Inc, Chicago, IL, USA). $p$-values less than 0.05 were considered statistically significant.

\section{Results}

The construction of slc10a2 overexpressed NSCLC cells

As shown in Fig. 1a, after $24 \mathrm{~h}$ treatment with pcDNA3.1-slc10a2 plasmid in $293 \mathrm{~T}$ cells, the $293 \mathrm{~T}$ cells had successfully transfected with slc10a2 gene, and efficiency of transfection is about $92 \%$. Then the supernatant of virus was added to A549 cells, after this slc10a2 overexpressed A549 cells were successfully constructed (Fig. 1b). Similarly we also used this method to construct slc10a2 overexpressed H1299 cells (data not show). The western blot results further demonstrated that the expression of slc10a2 was obviously higher in transfected group than control group (Fig. 1c). And the expression of slc10a2 was declined after treated with slc10a2-shRNA in A549 cells, this result suggests slc10a2-shRNA can effectively prohibit the expression of slc10a2 in A549 cells (Fig. 1d). In addition, slc10a2- 

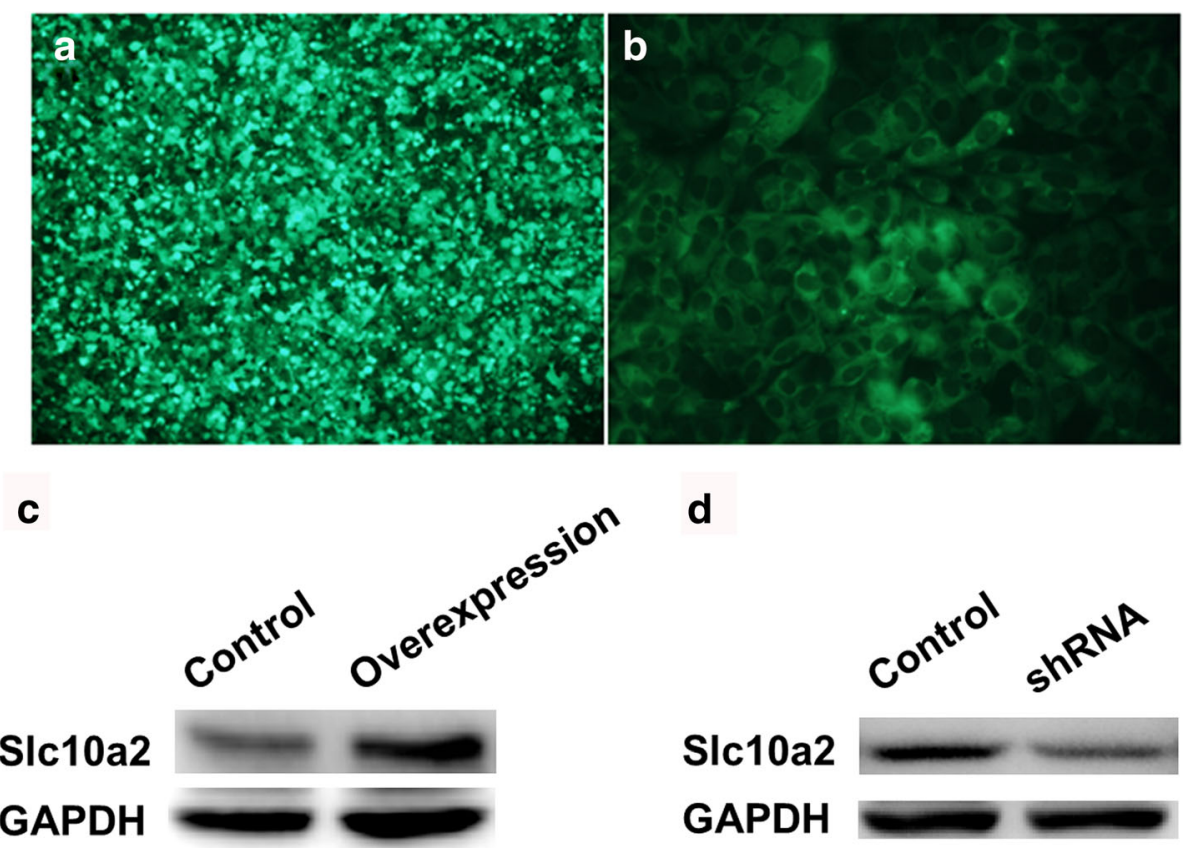

d

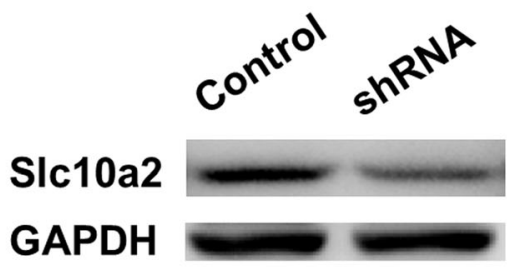

Fig. 1 The construction and identification of slc10a2 overexpressed A549 cells. a The immunofluorescence staining showed slc10a2 gene had successfully transfected into $293 \mathrm{~T}$ cells; b The immunofluorescence staining showed slc10a2 overexpressed A549 cells were successfully constructed; $\mathbf{c}$ The expression of slc10a2 was significant higher in slc10a2 overexpressed A549 cells than in A549 cells; d SIc10a2-shRNA can effectively inhibit the expression of slc10a2 in A549 cells. All experiments were repeated 3 times

shRNA also can significantly inhibit the expression of slc10a2 in H1299 cells (data not show).

\section{SIc10a2 plays an important role in the proliferation of} NSCLC cells with the treatment of bexarotene

As Fig. 2 showed, in comparison to the control group (without any treatment), bexarotene can inhibit the proliferation of A549 cells on day 3 and day 4. And the proliferation of slc10a2 overexpressed A549 cells was significantly prohibited with the treatment of bexarotene on both day 3 and day 4 . However, the proliferation of A549 cells was increased when co-treated with bexarotene and slc10a2-shRNA at all of the detected time points. Moreover, the same results can be seen

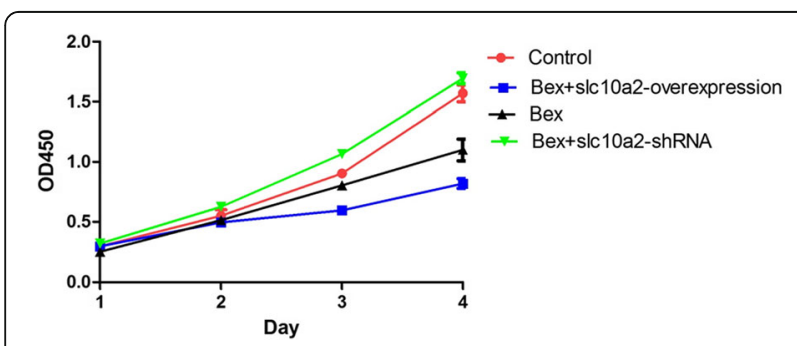

Fig. 2 The proliferation of A549 cells treated with bexarotene, bexarotene + slc10a2-shRNA, bexarotene + slc10a2-overexpression respectively. A549 cells without treatment as control. All experiments were repeated 3 times in H1299 cells (Additional file 1: Figure S1A). These results suggest slc10a2 involve in the process of bexarotene inhibits the proliferation of NSCLC cells.

Slc10a2 plays an important role in the invasion of NSCLC cells with the treatment of bexarotene

Transwell invasion test showed that (Fig. 3), the migration of A549 cells was decreased with the treatment of bexarotene for $24 \mathrm{~h}$ when compared with control group, the similar situation was discovered in slc10a2 overexpressed A549 cells treated with bexarotene. While the migration of A549 cells was increased when co-treated with bexarotene and slc10a2-shRNA. It reveals that slc10a2 involve in the process of bexarotene inhibits the invasion of NSCLC cells.

Slc10a2 plays an important role in the apoptosis of NSCLC cells with the treatment of bexarotene

As shown in Fig. 4a, in comparison to the control group (Fig. 4d), there is no significant difference in apoptosis rate between $0.1 \mathrm{mM}$ bexarotene treated group and control group. However, the apoptosis rate in $1 \mathrm{mM}$ and $10 \mathrm{mM}$ bexarotene treated groups was obviously higher than control group. In addition, the apoptosis rate of slc10a2 overexpressed A549 cells treated with $1 \mathrm{mM}$ and $10 \mathrm{mM}$ bexarotene was significantly increased than in 
a

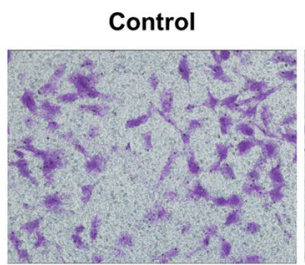

Bex + slc10a2-overexpression

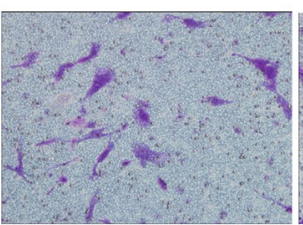

Bex

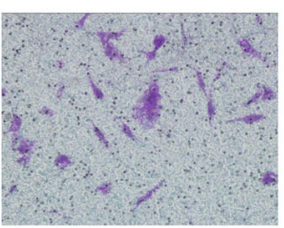

Bex +

slc10a2-shRNA

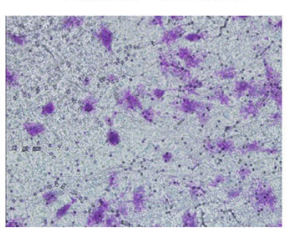

b

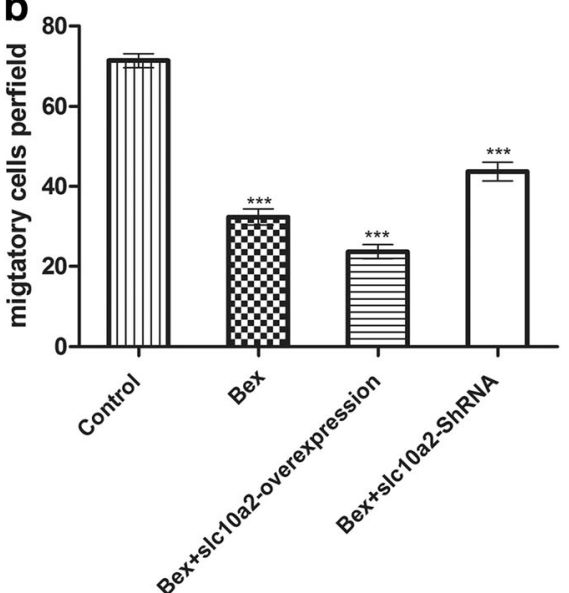

Fig. 3 The effects of slc10a2 on invasion of A549 cells treated with bexarotene. a The invasion behavior of A549 cells treated with bexarotene, bexarotene + slc10a2-shRNA, bexarotene + slc10a2-overexpression respectively, A549 cells without any treatment as control group. b The quantification of migratory A549 cells treated with bexarotene, bexarotene + slc10a2-shRNA, bexarotene + slc10a2-overexpression respectively. All experiments were repeated 3 times. ${ }^{* *} p<0.001$

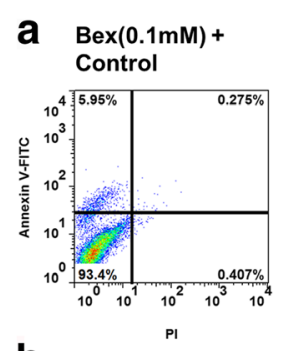

b

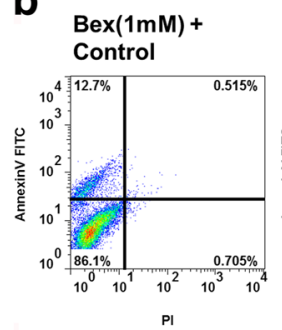

C
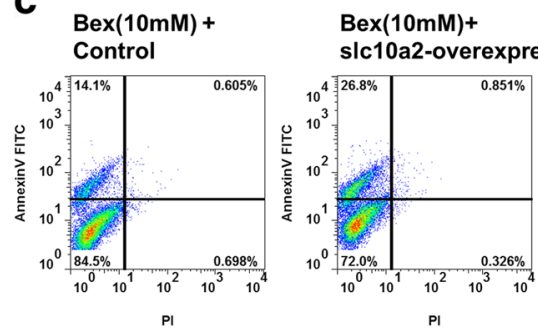

$\operatorname{Bex}(0.1 \mathrm{mM})+$ slc10a2-overexpression

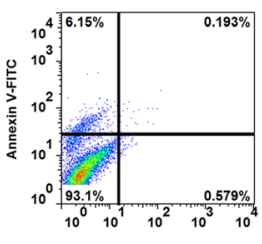

$\mathrm{PI}$

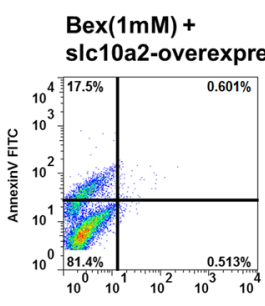

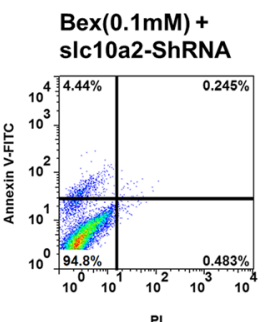

d
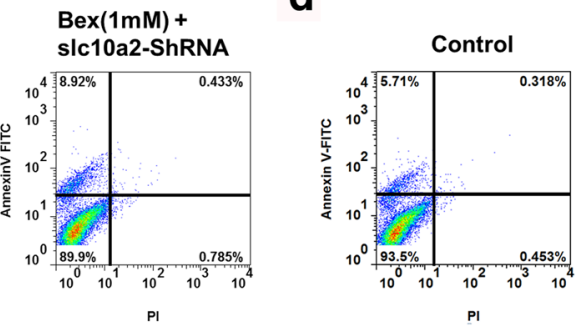

$\operatorname{Bex}(10 \mathrm{mM})+$

slc10a2-ShRNA

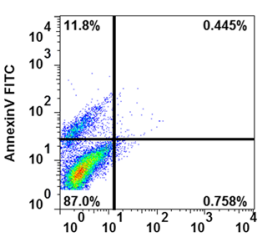

$\mathrm{PI}$

Fig. 4 The effects of slc10a2 on apoptosis of A549 cells treated with bexarotene. a The apoptosis rate of A549 cells treated with $0.1 \mathrm{mM}$ bexarotene, overexpressed slc10a2 in combination with $0.1 \mathrm{mM}$ bexarotene, slc10a2-shRNA in combination with $0.1 \mathrm{mM}$ bexarotene respectively; b The apoptosis rate of A549 cells treated with $1 \mathrm{mM}$ bexarotene, overexpressed slc10a2 in combination with $1 \mathrm{mM}$ bexarotene, slc10a2-shRNA in combination with $1 \mathrm{mM}$ bexarotene respectively; $\mathbf{c}$ The apoptosis rate of A549 cells treated with $10 \mathrm{mM}$ bexarotene, overexpressed slc10a2 in combination with $10 \mathrm{mM}$ bexarotene, slc10a2-shRNA in combination with $10 \mathrm{mM}$ bexarotene respectively, A549 cells without any treatment as control group (d). All experiments were repeated 3 times 

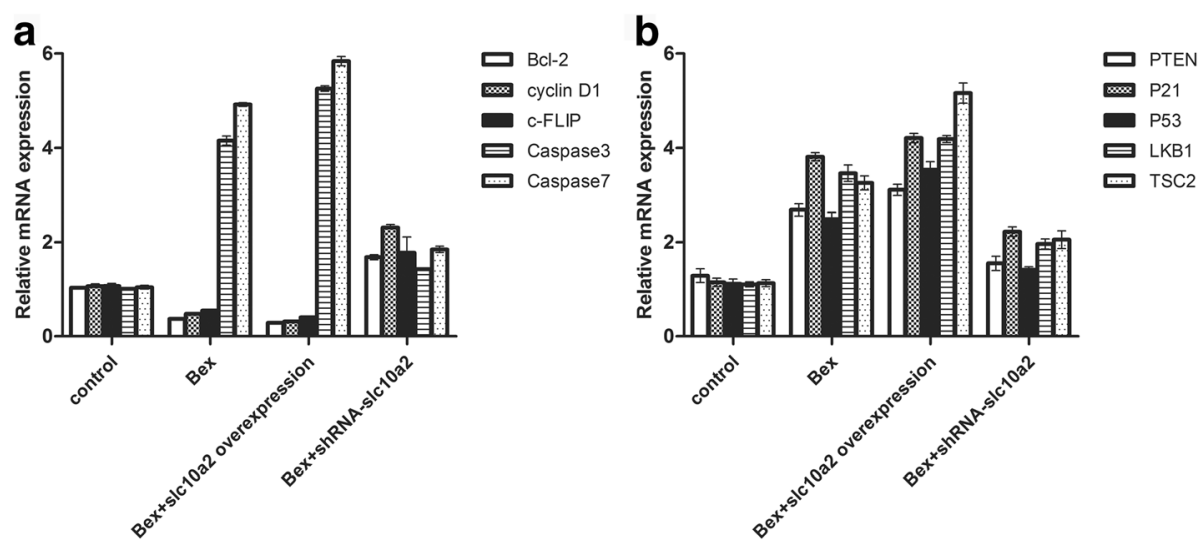

Fig. 5 The effects of slc10a2 on expression of apoptosis related genes in A549 cells treated with bexarotene. a The expression of apoptotic related genes Bcl-2, cyclin D1, c-FLIP, caspase 3, caspase 7 in A549 cells treated with bexarotene, overexpressed slc10a2 in combination with bexarotene, slc10a2-shRNA in combination with bexarotene respectively, A549 cells without any treatment as control group. $\mathbf{b}$ The expression of tumor suppressor genes PTEN, P21, P53, LKB1, TSC2 in A549 cells treated with bexarotene, overexpressed slc10a2 in combination with bexarotene, slc10a2-shRNA in combination with bexarotene respectively, A549 cells without any treatment as control group. All experiments were repeated 3 times

A549 cells, and it distinctly declined in A549 cells after treated with slc10a2-shRNA in combination with bexarotene when compared to the bexarotene single treated group (Fig. 4b and c). Moreover, the apoptosis of H1299 cells was significant increased when H1299 cells treated with $10 \mathrm{mM}$ bexarotene + slc10a2-overexpression, and it

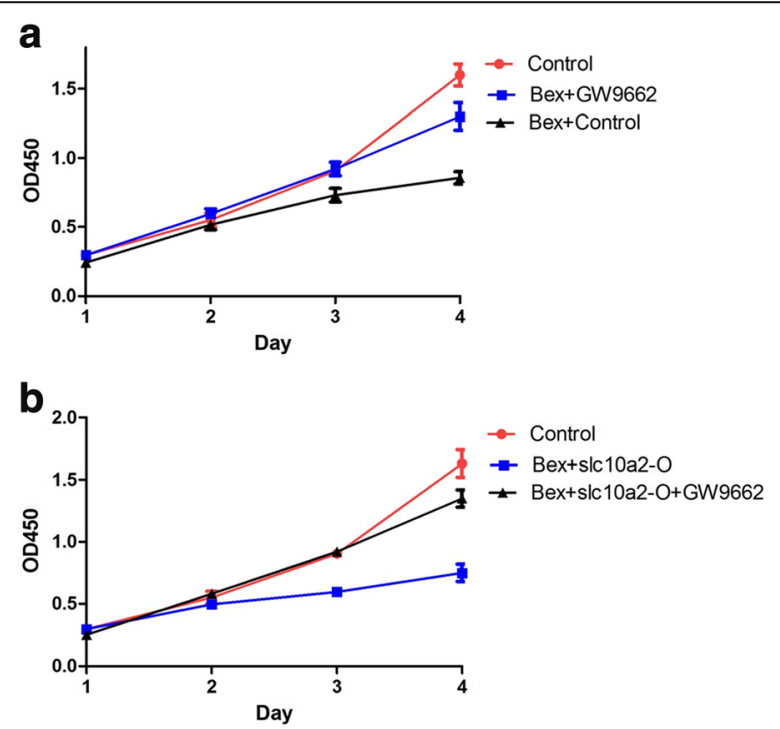

Fig. 6 Slc10a2 via PPARy plays an important role in the proliferation of A549 cells with the treatment of bexarotene. $\mathbf{a}$ The proliferation rate of A549 cells treated with bexarotene, bexarotene in combination with GW9662 respectively, A549 cells without any treatment as control group. $\mathbf{b}$ The proliferation rate of slc10a2 overexpressed A549 cells treated with bexarotene, bexarotene in combination with GW9662 respectively, A549 cells without any treatment as control group. All experiments were repeated 3 times decreased when H1299 cells treated with $10 \mathrm{mM}$ bexarotene + shRNA-slc10a2 (Additional file 1: Figure S1B).

\section{Slc10a2 plays an important role in tumor suppressor with the treatment of bexarotene}

As shown in Fig. 5 and Additional file 2: Figure S2, after A549 cells and H1299 cells treated with bexarotene for $24 \mathrm{~h}$, the expression of apoptotic related genes caspase 3, caspase 7, and tumor suppressor genes PTEN, P21, P53, LKB1 and TSC2 were significantly increased $(p<0.05)$ and the expression of anti-apoptotic genes bcl 2, cyclin D1, c-FLIP were decreased $(p<0.05)$. Similarly, the same situations were observed in slc10a 2 overexpressed A549 cells and H1299 cells. While, the expression of caspase 3, caspase 7, PTEN, P21, P53, LKB1 and TSC2 were reduced $(p<0.05)$ and the expression of bcl 2, cyclin D1, c-FLIP were increased $(p<0.05)$ when A549 cells and H1299 cells were co-treated with bexarotene and slc10a2-shRNA.

\section{SIc10a2 via PPARy plays an important role in the proliferation of NSCLC cells with the treatment of bexarotene}

According to the aforementioned, we have demonstrated that slc10a2 plays an important role in the proliferation of A549 cells with the treatment of bexarotene, how can the slc10a2 effect in this process. As Fig. 6a showed, by comparison, the proliferation of A549 cells was inhibited when treated with bexarotene on both day 3 and day 4 . However, we found that GW992 (selective PPAR $\gamma$ antagonist) can shortened the proliferative inhibition effects of bexarotene. The proliferation rate of A549 cells was higher in bexarotene in combination with GW9662 treated group than the bexarotene treated group on day 

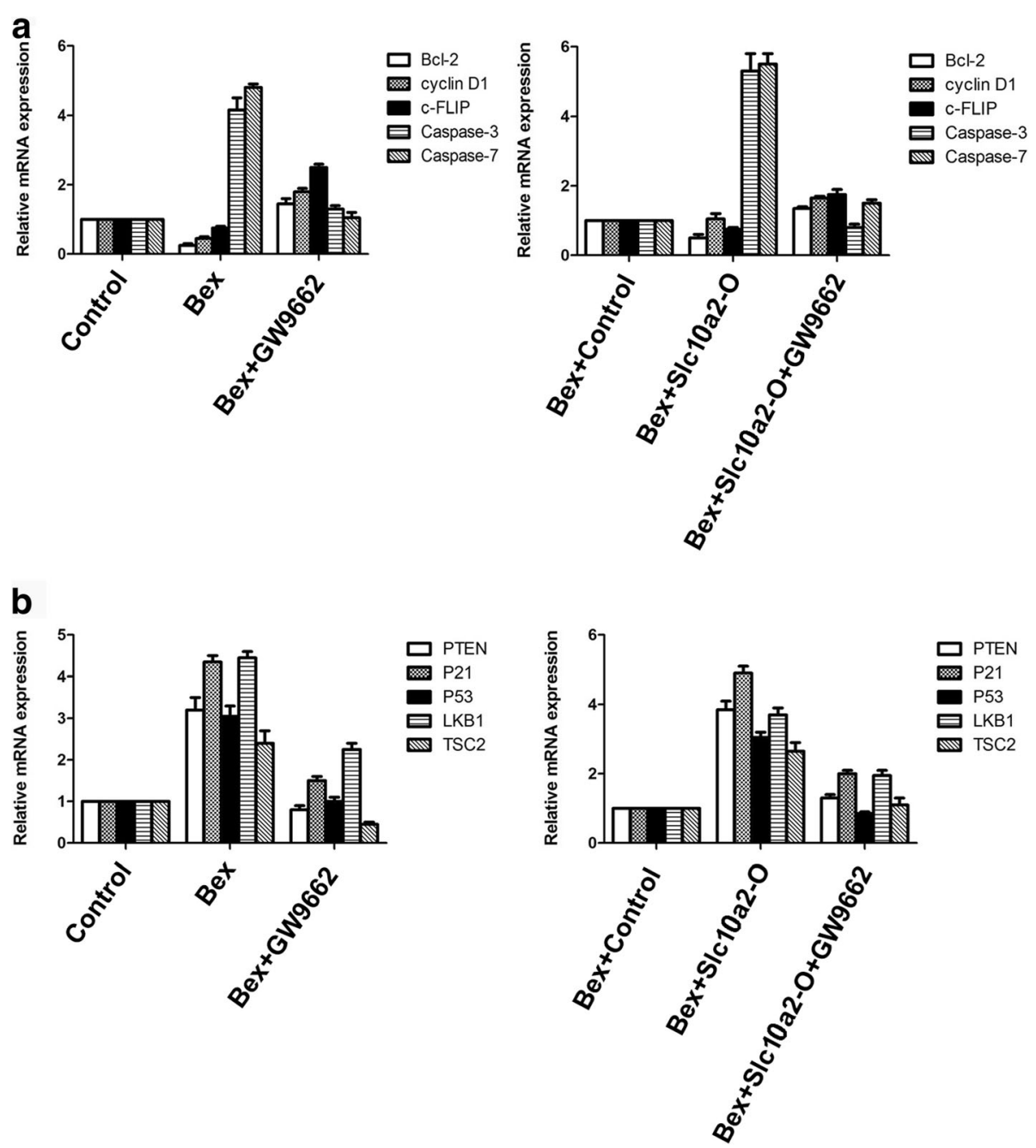

Fig. 7 SIc10a2 via PPARy plays an important role in tumor suppressor with the treatment of bexarotene. a The expression of apoptotic related genes Bcl-2, cyclin D1, c-FLIP, caspase 3, caspase 7 in A549 cells and slc10a2 overexpressed A549 cells when treated with bexarotene, bexarotene in combination with GW9662 respectively; b The expression of tumor suppressor genes PTEN, P21, P53, LKB1, TSC2 in A549 cells and slc10a2 overexpressed A549 cells when treated with bexarotene, bexarotene in combination with GW9662 respectively. A549 cells without any treatment as control group. All experiments were repeated 3 times

3 and day 4. Furthermore, a similar result was found in slc10a2 overexpressed A549 cells (Fig. 6b).

\section{Slc10a2 via PPARy plays an important role in tumor suppressor with the treatment of bexaroten}

We further explored whether slc10a2 via PPARy plays an important role in tumor suppressor with the treatment of bexarotene. As shown in Fig. 7 and Additional file 3: Figure S3 after A549 cells, H1299 cells or slc10a2 overexpressed A549 cells, H1299 cells treated with bexarotene, the expression of apoptotic genes caspase 3, caspase 7, and tumor suppressor genes PTEN, P21, P53, LKB1 and TSC2 were significantly increased $(p<0.05)$ and the expression of anti-apoptotic genes bcl 2, cyclin D1, c-FLIP were reduced $(p<0.05)$. While, the expression of caspase 3, caspase 7, PTEN, P21, P53, LKB1 and TSC2 were declined $(p<0.05)$ and the expression of bcl 2, cyclin D1, c-FLIP were increased $(p<0.05)$ when A549 cells, H1299 cells or slc10a2 overexpressed A549 cells, H1299 cells were co-treated with bexarotene and GW9662.

\section{Bexarotene inhibits the viability of NSCLC cells via slc10a2/PPARy/PTEN/mTOR signaling pathway}

As shown in Fig. 8a, b and Additional file 4: Figure S4, the western blotting and RT-PCR results showed the expression of slc10a2 was gradually enhanced with the increase of bexarotene's concentrations from $1 \mathrm{mM}$ to $10 \mathrm{mM}$. Also the expression of PPAR $\gamma$ was increased with the increase of bexarotene's concentrations. Additionally, the expression of slc10a2 can be reduced in Bex + GW9662 treated group. Moreover the expression of PTEN was increased in bexarotene treated group 


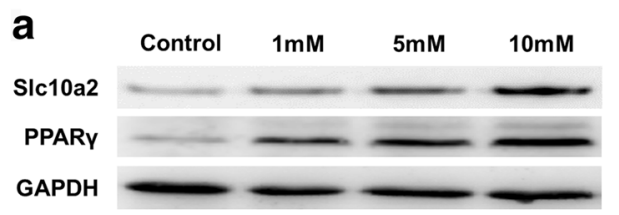

b

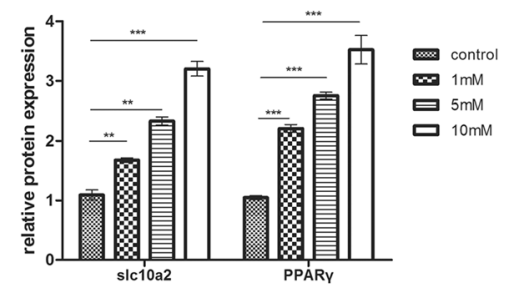

C

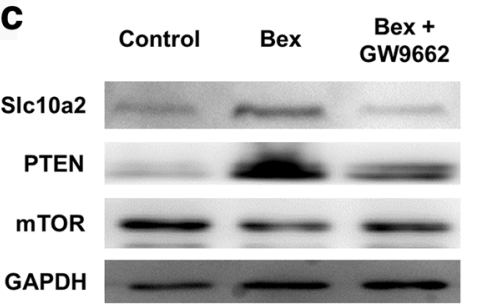

d

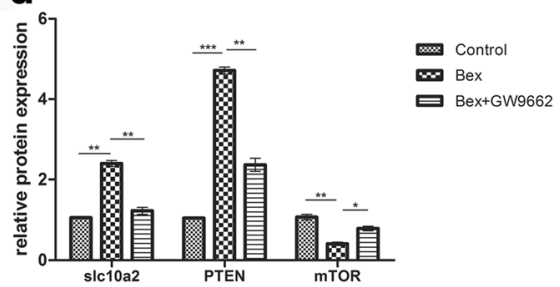

Fig. 8 Bexarotene inhibits the viability of A549 cells via slc10a2/PPARY/PTEN/mTOR signaling pathway. a The expression of slc10a2 and PPARY in A549 cells treated with 1 mM, 5 mM, 10 mM bexarotene respectively. b The quantification of slc10a2 and PPARy expression in A549 cells treated with 1 mM, 5 mM, 10 mM bexarotene respectively. c The expression of slc10a2, PTEN, mTOR in A549 cells treated with bexarotene, bexarotene + GW9662 respectively. $\mathbf{d}$ The quantification of slc10a2, PTEN, mTOR expression in A549 cells treated with bexarotene, bexarotene + GW9662. All experiments were repeated 3 times. ${ }^{*} p<0.05,{ }^{* *} p<0.01$

when compared to the control group, while it can be inhibit when A549 cells treated with bexarotene and GW9662. On the contrary, the expression of mTOR was suppressed by bexarotene, and this inhibition effects can be shortened by GW9662 (Fig. 8c, d).

\section{Discussion}

Three types of PPARs have been identified: alpha, gamma, and delta, they are a group of nuclear receptor proteins which function as transcription factors regulating the expression of genes [19]. Among the three phenotypes, PPAR $\gamma$ has been attracting tremendous attention, the previous studies revealed that PPAR $\gamma$ plays essential roles in the regulation of cellular differentiation, development, and metabolism (carbohydrate, lipid, protein), and tumorigenesis [20, 21].

A mass of studies have demonstrated that PPARY agonists via inhibiting the expression of cyclinD1, cyclinB, cyclinE, CDK4 and CDK2 and increasing the expression of CDKN1A to prevent cell cycle from $\mathrm{G} 1$ to $\mathrm{S}$ phase to prohibit tumor cells proliferation [22-26]. In this study we found that bexarotene worked as a PPAR $\gamma$ agonists, which was capable of enhancing the expression of PPAR $\gamma$, then the expression of cyclinD1 was suppressed and the proliferation of A549 cells was prohibited, these results were consistent with the previous studies.

The activation of PPAR $\gamma$ can induce tumor cell apoptosis via several different pathways. PPAR $\gamma$ agonists was able to up-regulate pro-apoptotic protein BAX and BAD expression, and then induced glioma cells apoptosis through releasing cytochrome $\mathrm{C}$ and activating the activation of caspase [27]. Li et al. reported the activation of
PPAR $y$ was associated with a decrease of the expression of Bcl-2 and increase of the expression of P53 in human melanoma cell line A375 cells [28]. Similarly, we found that the expression of anti-apoptotic proteins $\mathrm{Bcl}-2$, cyclin D1 and c-FLIP was reduced whereas the expression of apoptotic proteins caspase-3, caspase-7 and tumor suppressor gene PTEN, P21, P53, LKB1, TSC2 were accelerated in A549 cells with the treatment of bexarotene, which was associated with the activation of PPAR $\gamma$ through enhancing the expression of slc10a2, resulting in promoting the apoptosis of A549 cells.

Moreover, the activation of PPARy can reduce the invasion ability of tumor cells. Thiazolidinedione (TZD) is a synthetic agonist of PPAR $\gamma$, which contains troglitazone, pioglitazone and rosiglitazone [29]. Willson et al. discovered that after adrenocortical cancer cell lines H295R cells co-cultured with pioglitazone and rosiglitazone, the expression of MMP-2 which play an important role in cell migration was reduced, and the migration of H295R cells was significantly declined [30]. Also Galli et al. found that TZD can effectively inhibit tumor cell invasion, after pancreatic cancer cell treated with TZD for $24 \mathrm{~h}$, the activity and transcriptional level of MMP-2 were declined [31]. And in this study, the migration ability of A549 cells was significantly shortened after treated with bexarotene, whereas the migration of A549 cells was distinctly accelerated after with the treatment of bexarotene in combination with GW9662.

PI3K/Akt/mTOR signaling pathway exists in almost all mammals, it regulates cell growth mainly through controlling the protein synthesis. PTEN as a negative regulator of this pathway via suppressing the expression of 
PI3K and Akt. Patel et al. revealed that PPAR $\gamma$ can combined with peroxisome proliferator responsive element 1 (PPRE1) and PPRE2, the upstream gene of PTEN, and increased the expression of PTEN then induced phosphorylation of Akt decreased, cells differentiation and apoptosis [32]. And it has been preclinically showed that deficiency of TSC2 or PTEN expression induces impaired PI3K/Akt/mTOR activation, suggesting that mTOR overexpression with the loss of PTEN plays a key role in the development and progression of pancreatic neuroendocrine tumors [33]. In this study, we discovered that bexarotene accelerated the expression of PPARy through enhancing the expression of slc10a2, also the expression of PPAR $\gamma$ was promoted, while the expression of mTOR was declined, thus the viability of A549 cells was suppressed. Finally this study has some limitations, for example, PPAR agonist bexarotene induces PPARy and slc10a2 in a dose dependent manner, but the effects of bexarotene on PPAR $\alpha, \operatorname{PPAR} \beta / \delta$ expression we don't explore. We have clarified limitations in the discussion section, and we will explore the effects of bexarotene on PPAR $\alpha$, PPAR $\beta / \delta$ expression in our future study.

\section{Conclusion}

In this study we found that bexarotene can suppress the proliferation, migration, and promote the apoptosis of NSCLC cells. Moreover, we demonstrated that this effects owned to the increased expression of PPAR $\gamma$ via enhancing the expression of slc10a2, then up-regulated the expression of PTEN and down-regulated the expression of mTOR, thus increased the expression of apoptotic genes and anti-cancer genes, and reduced the expression of anti-apoptotic genes to suppress the proliferation of NSCLC cells and promote the apoptosis of NSCLC cells [34].

\section{Additional files}

Additional file 1: Figure S1. (A) The proliferation of $\mathrm{H} 1299$ cells treated with bexarotene, bexarotene + shRNA-slc10a2, bexarotene + slc10a2overexpression respectively, H1299 cells without treatment as control. (B) The apoptosis of $\mathrm{H} 1299$ cells treated with bexarotene, bexarotene + shRNA-slc10a2, bexarotene + slc10a2-overexpression respectively, H1299 cells without treatment as control. All experiments were repeated 3 times. (TIFF $739 \mathrm{~kb}$ )

Additional file 2: Figure S2. (A) The expression of apoptotic related genes Bcl-2, cyclin D1, c-FLIP, caspase 3, caspase 7 in H1299 cells treated with bexarotene, overexpressed slc10a2 in combination with bexarotene, slc10a2-shRNA in combination with bexarotene respectively. (B) The expression of tumor suppressor genes PTEN, P21, P53, LKB1, TSC2 in H1299 cells treated with bexarotene, overexpressed slc10a2 in combination with bexarotene, slc10a2-shRNA in combination with bexarotene respectively, H1299 cells without any treatment as control group. All experiments were repeated 3 times. (TIFF $516 \mathrm{~kb}$ )

Additional file 3: Figure S3. (A) The expression of apoptotic related genes Bcl-2, cyclin D1, c-FLIP, caspase 3, caspase 7 in H1299 cells when treated with bexarotene, bexarotene in combination with GW9662 respectively. (B) The expression of apoptotic related genes BCl-2, cyclin D1, c-FLIP, caspase 3, caspase 7 in slc10a2 overexpressed H1299 cells when treated with bexarotene, bexarotene in combination with GW9662 respectively. (C) The expression of tumor suppressor genes PTEN, P21, P53, LKB1, TSC2 in H1299 cells when treated with bexarotene, bexarotene in combination with GW9662 respectively. (D) The expression of tumor suppressor genes PTEN, P21, P53, LKB1, TSC2 in slc10a2 overexpressed H1299 cells when treated with bexarotene, bexarotene in combination with GW9662 respectively. H1299 cells without any treatment as control group. All experiments were repeated 3 times. (TIFF $882 \mathrm{~kb}$ )

Additional file 4: Figure S4. The expression of slc10a2 in A549 cells treated with $1 \mathrm{mM}, 5 \mathrm{mM}, 10 \mathrm{mM}$ bexarotene respectively, A549 cell without treatment as control. (TIFF $68 \mathrm{~kb}$ )

\section{Abbreviations}

ANOVA: One-way analysis of variance; CCK-8: Cell Counting Kit-8; HDL: Highdensity lipoprotein; NSCLC: Non-small cell lung cancer; PPAR: Peroxisome proliferator-activated receptor; PPRE: Peroxisome proliferator responsive element; RXRs: Retinoid X receptors; TZD: Thiazolidinedione

\section{Acknowledgements}

Not applicable

\section{Funding}

This work was supported by Major Key Project of Shanghai Chest Hospital Science \& Technology foundation (2014YZDC10600). The funding source has no role in the study design, analysis, or interpretation of the data, writing of the manuscript, or the decision to submit the manuscript for publication.

\section{Availability of data and materials}

The data and materials used in this current study are available from the corresponding author on reasonable request.

\section{Authors' contributions}

Prof. SL designed this study; Prof. YST gave final approval of the version to be published; XHA wrote and revised this paper; FM made substantial contributions to acquisition of data, analysis and interpretation of data, SPS and JJW performed experiments and collected data. All authors have read and approved the manuscript.

\section{Ethics approval and consent to participate}

Not applicable

\section{Consent for publication}

Not applicable

Competing interests

The authors declare that they have no competing interests.

\section{Publisher's Note}

Springer Nature remains neutral with regard to jurisdictional claims in published maps and institutional affiliations.

Received: 2 December 2015 Accepted: 13 March 2018

Published online: 11 April 2018

\section{References}

1. Zheng R, Zeng H, Zhang S, Fan Y, Qiao Y, Zhou Q, et al. Lung cancer incidence and mortality in China, 2010. Thoracic Cancer. 2016;7(1):94-99.

2. Hermann TW, Yen W-C, Tooker P, Fan B, Roegner K, Negro-Vilar A, et al. The retinoid $X$ receptor agonist bexarotene (Targretin) synergistically enhances the growth inhibitory activity of cytotoxic drugs in non-small cell lung cancer cells. Lung Cancer. 2005:50:9-18.

3. Rizvi NA, Marshall JL, Dahut W, Ness E, Truglia JA, Loewen G, et al. A phase I study of LGD1069 in adults with advanced cancer. Clin Cancer Res. 1999;5:1658-64. 
4. Miller VA, Benedetti FM, Rigas JR, Verret AL, Pfister DG, Straus D, et al. Initial clinical trial of a selective retinoid X receptor ligand, LGD1069. J Clin Oncol. 1997;15:790-5.

5. Luo W, Schork NJ, Marschke KB, Ng S-C, Hermann TW, Zhang J, et al. Identification of polymorphisms associated with hypertriglyceridemia and prolonged survival induced by bexarotene in treating non-small cell lung cancer. Anticancer Res. 2011;31:2303-11.

6. Blumenschein GR, Khuri FR, von Pawel J, Gatzemeier U, Miller WH, Jotte RM, et al. Phase III trial comparing carboplatin, paclitaxel, and bexarotene with carboplatin and paclitaxel in chemotherapy-naive patients with advanced or metastatic non-small-cell lung cancer: SPIRIT II. J Clin Oncol. 2008;26:1879-85.

7. Malik SM, Collins B, Pishvaian M, Ramzi P, Marshall J, Hwang J. A phase I trial of bexarotene in combination with docetaxel in patients with advanced solid tumors. Clin Lung Cancer. 2011;12:231-6.

8. Tang X-H, Gudas LJ. Retinoids, retinoic acid receptors, and cancer. Annu Rev Pathol. 2011;6:345-64.

9. Dawson Ml, Xia Z. The retinoid X receptors and their ligands. Biochim Biophys Acta. 2012;1821:21-56.

10. Brabender J, Metzger R, Salonga D, Danenberg KD, Danenberg PV, Hölscher $\mathrm{AH}$, et al. Comprehensive expression analysis of retinoid acid receptors and retinoid $X$ receptors in non-small cell lung cancer: implications for tumor development and prognosis. Carcinogenesis. 2005;26(3):525-30.

11. Wong MH, Rao PN, Pettenati MJ, Dawson PA. Localization of the ileal sodium-bile acid cotransporter gene (SLC10A2) to human chromosome 13q33. Genomics. 1996;33:538-40.

12. Dawson PA. Role of the intestinal bile acid transporters in bile acid and drug disposition. Drug transporters: Springer; Handb Exp Pharmacol. 2011; (201):169-203.

13. Kosters A, Karpen SJ. Bile acid transporters in health and disease. Xenobiotica. 2008;38:1043-71.

14. Paresh PC, Peter WS. Resveratrol promotes degradation of the human bile acid transporter ASBT (SLC10A2). Biochem J. 2014;459:301-12.

15. Kitayama K, Nakai D, Kono K, van der Hoop AG, Kurata $H$, de Wit EC, et al. Novel non-systemic inhibitor of ileal apical $\mathrm{Na}<$ sup $>+</$ sup $>-$ dependent bile acid transporter reduces serum cholesterol levels in hamsters and monkeys. Eur J Pharmacol. 2006;539:89-98.

16. West KL, McGrane M, Odom D, Keller B, Fernandez ML. SC-435, an ileal apical sodium-codependent bile acid transporter inhibitor alters mRNA levels and enzyme activities of selected genes involved in hepatic cholesterol and lipoprotein metabolism in Guinea pigs. J Nutr Biochem. 2005; 16:722-8

17. Liu ZY, Wang JY, Liu HH, Ma XM, Wang CL, Zhang XP, et al. Retinoblastoma protein-interacting zinc-finger gene 1 (RIZ1) dysregulation in human malignant meningiomas. Oncogene. 2013;32:1216.

18. Qiu Z, Dyer KD, Xie Z, Rådinger M, Rosenberg HF. GATA transcription factors regulate the expression of the human eosinophil-derived neurotoxin (RNase 2) gene. J Biol Chem. 2009;284:13099-109.

19. Michalik L, Auwerx J, Berger JP, Chatterjee VK, Glass CK, Gonzalez FJ, et al. International Union of Pharmacology. LXI. Peroxisome proliferator-activated receptors. Pharmacol Rev. 2006;58:726-41.

20. Berger J, Moller DE. The mechanisms of action of PPARs. Annu Rev Med 2002;53:409-35.

21. Feige JN, Gelman L, Michalik L, Desvergne B, Wahli W. From molecular action to physiological outputs: peroxisome proliferator-activated receptors are nuclear receptors at the crossroads of key cellular functions. Prog Lipid Res. 2006;45:120-59.

22. Nagamine M, Okumura T, Tanno S, Sawamukai M, Motomura W, Takahashi $\mathrm{N}$, et al. PPARy ligand-induced apoptosis through a p53-dependent mechanism in human gastric cancer cells. Cancer Sci. 2003;94:338-43.

23. Baek SJ, Wilson LC, Hsi LC, Eling TE. Troglitazone, a peroxisome proliferatoractivated receptor $Y$ (PPARY) ligand, selectively induces the early growth Response-1 gene independently of PPARY. A novel mechanism for its antitumorigenic activity. J Biol Chem. 2003;278:5845-53.

24. Ricote M, Li AC, Willson TM, Kelly CJ, Glass CK. The peroxisome proliferatoractivated receptor- $\gamma$ is a negative regulator of macrophage activation. Nature. 1998:391:79-82.

25. Han S, Sidell N, Fisher PB, Roman J. Up-regulation of p21 gene expression by peroxisome proliferator-activated receptor $\gamma$ in human lung carcinoma cells. Clin Cancer Res. 2004;10:1911-9.

26. Koga H, Sakisaka S, Harada M, Takagi T, Hanada S, Taniguchi E, et al. Involvement of p21WAF1/Cip1, p27Kip1, and p18INK4C in troglitazone- induced cell-cycle arrest in human hepatoma cell lines. Hepatology. 2001; 33:1087-97.

27. Zander T, Kraus JA, Grommes C, Schlegel U, Feinstein D, Klockgether T, et al. Induction of apoptosis in human and rat glioma by agonists of the nuclear receptor PPARY. J Neurochem. 2002;81:1052-60.

28. Li Y, Meng Y, Li H, Li J, Fu J, Liu Y, et al. Growth inhibition and differentiation induced by peroxisome proliferator activated receptor gamma ligand rosiglitazone in human melanoma cell line A375. Med Oncol. 2006;23:393-402.

29. Lehmann JM, Moore LB, Smith-Oliver TA, Wilkison WO, Willson TM, Kliewer SA. An antidiabetic thiazolidinedione is a high affinity ligand for peroxisome proliferator-activated receptor Y (PPARY). J Biol Chem. 1995;270:12953-6.

30. Ferruzzi P, Ceni E, Tarocchi M, Grappone C, Milani S, Galli A, et al. Thiazolidinediones inhibit growth and invasiveness of the human adrenocortical cancer cell line H295R. J Clin Endocrinol Metab. 2005:90:1332-9.

31. Galli A, Ceni E, Crabb DW, Mello T, Salzano R, Grappone C, et al. Antidiabetic thiazolidinediones inhibit invasiveness of pancreatic cancer cells via PPARY independent mechanisms. Gut. 2004;53:1688-97.

32. Patel L, Pass I, Coxon P, Downes CP, Smith SA, Macphee CH. Tumor suppressor and anti-inflammatory actions of PPARY agonists are mediated via upregulation of PTEN. Curr Biol. 2001;11:764-8.

33. Han $X, J i Y$, Zhao J, Xu X, Lou W. Expression of PTEN and mTOR in pancreatic neuroendocrine tumors. Tumor Biol. 2013;34:2871-9.

34. Ai X, Lu S. PUB061 Bexarotene inhibits the viability of A549 cells via slc10a2/ PPARy/PTEN/mTOR signaling pathway. J Thorac Oncol. 2015;10:761-90.

\section{Submit your next manuscript to BioMed Central and we will help you at every step:}

- We accept pre-submission inquiries

- Our selector tool helps you to find the most relevant journal

- We provide round the clock customer support

- Convenient online submission

- Thorough peer review

- Inclusion in PubMed and all major indexing services

- Maximum visibility for your research

Submit your manuscript at www.biomedcentral.com/submit

) Biomed Central 